\title{
El TRADE MARKETING EN LA EVOLUCIÓN COMERCIAL DE LA EMPRESa Grupo el Rosado de Ecuador
}

\author{
German Jazmany Zambrano Verdesoto ${ }^{1}$
}

Recibido: 15/09/2020 • Aceptado: 18/10/2021

\begin{abstract}
Cómo citar: Zambrano Verdesoto, G. J. (2021). El trade marketing en la evolución comercial de la empresa Grupo el Rosado de Ecuador. Ciencia, Economía y Negocios, 5(2), 117-118. Doi: https://doi.org/10.22206/ ceyn.2021.v5i2.pp117-118
\end{abstract}

\section{Resumen}

El sistema comercial ha evoluciona en su forma de hacer negocios, para incrementar su accionar en los procesos de ventas y distribución ha utilizado diferentes estrategias para llegar a los clientes satisfactoriamente. El presente trabajo tiene como objetivo fundamentar la importación de utilizar el trade marketing en el sistema comercial tomando como ejemplo la empresa Grupo el Rosado en su evolución comercial en Ecuador. Para esta investigación se utilizó un estudio bibliográfico y documental de artículos científicos, tesis, libros y periódicos, publicados en base de datos virtuales y físicas de carácter especializados en marketing, lo cual forma un aporte explicativo, basado en un estudio inductivo partiendo de lo general al análisis teórico. Se concluye que el trade marketing permite a las empresas tener un crecimiento vertiginoso en el sistema comercial que permite fundamentar con diferentes estrategias como, merchandising, gestión de categorias por productos, promociones, programas de recompensas, estrategias de segmentación basadas en el comportamiento de compra, al desarrollar estas estrategias comerciales las empresas logran tener un crecimiento significativo en los diferentes segmentos de mercados para generar satisfacción del cliente. Finalmente, se plantea que las empresas que utilizan el trade marketing optimicen la logistica y mejoren las ventas para generar una cadena de valor que este interrelacionado con los clientes.

Palabras clave: clientes, distribución, estrategias, merchandising, segmentación.

\footnotetext{
${ }^{1}$ Instituto Superior Universitario Bolivariano de Tecnología, Ecuador. ORCID: 0000-0002-1642-2799, Correo-e: eduactitud@gmail.com
}

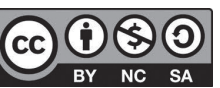




\title{
Trade MARKETING APPLIED IN THE DEVELOPMENT OF ECUADOR'S BUSINESS GROUP ROSADO
}

\author{
German Jazmany Zambrano Verdesoto \\ Received: $\mathrm{xxx} \bullet$ Approved: $\mathrm{xxx}$
}

\begin{abstract}
The commercial system has evolved in the way of doing business, to increase its actions in the sales and distribution processes, it has used different strategies to successfully reach customers. The objective of this work is to support the importation of using trade marketing in the commercial system, taking the Grupo el Rosado company as an example in its commercial evolution in Ecuador. For this research, a bibliographic and documentary study of scientific articles, theses, books and newspapers, published in virtual and physical databases specialized in marketing, was used, which forms an explanatory contribution, based on an inductive study based on the general to theoretical analysis. It is concluded that trade marketing allows companies to have a vertiginous growth in the commercial system that allows to base with different strategies such as merchandising, product category management, promotions, reward programs, segmentation strategies based on purchasing behavior, By developing these commercial strategies, companies achieve significant growth in different market segments to generate customer satisfaction. Finally, it is proposed that companies that use trade marketing optimize logistics and improve sales to generate a value chain that is interrelated with customers.
\end{abstract}

Keywords: customers, distribution, strategies, segmentation, merchandising. 


\section{Introducción}

El desarrollo del marketing ha evolucionado en los diferentes procesos comerciales, su fundamento está dado en establecer relaciones con los clientes basado en las necesidades que tiene el mercado. En los estudios realizados referente al marketing se ha establecido diferentes estrategias que mejoren el sistema comercial. Kotler y Armstrong (2013) describe. “ El desarrollo del marketing se basa en establecer relaciones redituables que perduren y se solidifiquen una interrelación entre cliente y la empresa" (p.5). Estos autores tienen gran renombre en el marketing y son parte fundamental de la evolución de este, en sus estudios han de proponer diferentes conceptos basados en experiencias prácticas con empresas donde se han implementado diferentes estrategias que permitan el desarrollo comercial de los productos o servicios de manera eficiente.

Las empresas actualmente desean implementar el marketing con un enfoque social que se basa en contribuir parte de sus ganancias en diferentes programas que permitan mejorar las condiciones de vida de las personas, por ejemplo, el marketing social es aplicado por grandes empresas de Ecuador, estas generan beneficios a diferentes sectores vulnerables con becas para estudios. En este proceso las empresas planifican estrategias de marketing que se enfoquen en destacar las marcas utilizando estrategias de posicionamiento que permitan captar mayor participación del mercado.

El trade marketing siempre ha estado presente en las empresas y estas tienen diferentes formas de utilizarlo como una herramienta para comercializar sus producto o servicio de manera eficiente utilizando los diferentes canales de distribución. "Un canal de distribución es la ruta que toman los bienes durante el proceso de ventas desde el proveedor hasta el cliente" (Jobber y Lancaster, 2012, p.119). Un canal es la base primordial para que el producto llegue de manera satisfactoria al consumidor final, desde que el producto es fabricado para por diferentes procesos hasta llegar al canal detallista o minorista que son los encargados en vender el producto al consumidor final, en este proceso el canal intermediario son los encargados de distribuir los productos y también de generar funciones básicas que permitan investigar el mercado para comercializar 
el producto y tiene como prioridad crear estrategias de promociones hacia el consumidor utilizando medios de comunicación para enfocar la comercialización de los productos o servicios.

\section{Análisis de la evolución del trade marketing de la empresa Grupo el Rosado}

El trade marketing ha evolucionado junto con el dinamismo económico que tiene cada país, este desarrollo tiene una estrecha relación entre el marketing y las ventas la primera está relacionada a desarrollar estrategias que permitan el posicionamiento de la empresa para promocionar productos que se comercializan en el mercado y las ventas tiene una estrecha relación con las diferentes promociones que se realiza y el consumidor final pueda comprar un producto.

Esta investigación es bibliográfica y se basa en un estudio referente al trade marketing en la empresa Grupo el Rosado de Ecuador. "Inicia sus actividades en el año 1936 por un emprendedor Don Alfredo Czarninski que inicia con una pequeña pastelería ubicada en la ciudad de Guayaquil tradicionalmente ubicada en la avenida nueve de octubre en este desarrollo cambio un nuevo concepto de restaurante en el cual tuvo una gran aceptación en mercado y desarrollaron en los consumidores variedad en sus servicios de comida" (Grupo el Rosado, s.f.). En este proceso de desarrollo comercial que tenía la ciudad de Guayaquil la empresa también incursiona en nuevos mercados incluyendo un almacén de variedades para el hogar ubicado al frente del restaurante, este almacén se enfoca en la línea de negocio de venta víveres y diferentes artículos para el hogar.

En este proceso de desarrollo la empresa crea un supermercado El Rosado la cual establece un sistema de ventas utilizando como modelos los auto servicios en diferentes sectores del país siendo pioneros en este tipo ventas. Al tener un crecimiento acelerado en este modelo de negocio inicia a generar nuevas plazas de trabajo, en este proceso de innovación acelerado en el año 1958 le da el nombre de Mi Comisariato bajo el modelo de hipermarket ubicados en diferentes ciudades del Ecuador entre las ciudades que más presencia tiene es en Guayaquil y Quito, al ser pioneros en procesos de innovación en el mercado local han desarrollado tres líneas de productos que son: 


\subsection{Línea de negocio Retail}

Mi comisariato: actualmente posee 46 establecimientos a nivel nacional es una de la cadena de supermercados más relevantes del país y tiene diferentes marcas de importación que permite distribuir diferentes marcas como Florida's Natural, Betis y también licores como vinos, cerveza royal dutch, también cuenta con una marca propia nacionales en víveres, bebidas, productos de limpiezas que compiten con otras marcas reconocidas en el mercado, su principal estrategia se fundamenta en vender sus productos a menor precio.

1. Mi panadería: tiene 39 locales a nivel nacional tiene su propia plata panificadora en la que se encarga de distribuir a diario a los supermercados del país, cuenta con su propio sistema de gestionar sus productos al consumidor final.

2. Mini: son establecimientos pequeños que se encuentra estratégicamente en diferentes ciudades del país, estos locales ofrecen productos específicos que se caracterizan por tener miniprecios para los consumidores finales.

3. Ferrisariato: es una de las cadenas más grande del país con 37 locales a nivel nacional y ofrece variedad de artículos de ferretería, madera, mampostería y también cuenta con varios productos como muebles oficina y hogar, cuenta con marcas propias como Tekno que tiene líneas de audio y video.

4. Mi juguetería: es una de las cadenas de artículos para nińos cuenta con 37 locales a nivel nacional, sus principales marcas que comercializa son Mattel y Hasbro, su principal estrategia está basada en las experiencias que los consumidores tienen en el establecimiento.

5. Rio Store: tiene 33 locales a nivel nacional está línea está dedicada a la venta de ropa, perfumería, maquillaje, tienes diferentes secciones para ropa nińos y adultos.

6. Baby center: es una tienda segmentada para el cuidado de niños de cero a dos años la cual tiene gran variedad de ropa y artículos para bebé.

7. Metrópolis: es una tienda especializada en equipos de audio y video, es el principal distribuidor de productos de Apple en Ecuador.

8. Hipermarket: tiene 26 locales en Ecuador en donde sus clientes encuentran las diferentes líneas que comercializa sus productos, en 
estos hipermarket encuentran marcas de ferrisariato, rio store, $\mathrm{mi}$ juguetería, esto permite a los clientes cubrir sus principales demandas de comprar, este establecimiento cuenta un área de hasta 22.000 metros cuadrados para cada línea de negocio.

\subsection{Línea de negocio entretenimiento}

1. Riocentro Shopping: existen 5 centro comerciales en el país la cual le presenta a sus clientes una gama de productos y servicios, cuenta con salas de cine, patios de comidas supermercados y locales de ropa.

2. Paseo Shopping: existen 14 comerciales que están ubicados en diferentes ciudades del país brindado una variedad de productos y servicios al consumidor final dando como prioridad que todo lo que compren es a precios bajos y de calidad.

3. Supercines: cuenta con 22 establecimiento y 174 salas de cines siendo pionero en el mercado hay salas que tiene gama tecnológica sofisticadas con salas GT MAX que son las más grandes del mundo.

4. Radio disney: es una emisora radial su principal atractivo son la interacción de promociones y artistas invitados que tiene el radio oyente.

\subsection{Líneas de restaurantes}

1. Carl's Jr: es parte del Grupo el Rosado cuenta con 20 restaurantes a nivel nacional su origen es California EE. UU. su principal producto son la hamburguesa al carbón.

2. Chili's: tiene 8 locales a nivel nacional es también una franquicia internacional de origen EE. UU., su principal especialidad son las comidas a la parrilla, y su función es un bar restaurante.

3. Red Lobster: es una franquicia EE. UU. su carta de presentación son la comida de marisco.

Estas tres líneas de negocios como el retail, entretenimiento y restaurante estructuran la empresa Grupo el Rosado que brinda a sus clientes una gama espectacular de productos y servicios para todos los gustos abarcando con el $100 \%$ de las necesidades que tienen los clientes. La evolución que ha tenido la empresa que nace como pastelería hace 83 años y ahora se convierte en una de las empresas pioneras en el crecimiento 
económico en Ecuador estableciendo un modelo de negocio vertiginoso y que su crecimiento tanto en infraestructura comercial, física y tecnológica han ido creciendo, a pesar de las diferentes situaciones políticas y económicas desfavorables que ha tenido el país su pilar fundamental se basa en los principales líneas de negocio que ha creado, y esto ha generado que la empresa genere un compromiso social generando fuentes de trabajos de miles de personas en el país.

\section{Estrategias del trade marketing su relación y efectividad en el Grupo el Rosado}

Las estrategias de marketing están interrelacionadas con el trade ya que su fuente primordial para establecer alguna estrategia está basada en las necesidades de establecer una relación con los clientes basándose en diferentes productos que se comercializan tanto en cadenas minoristas o grandes supermercados. Carrillo (2017) en su estudio realizado se enfoca en establecer vínculos rentables con los clientes a través del uso eficiente en el proceso de distribución. Ferrel y Hartiline (2012) describe "Es muy importante la distribución para que los compradores puedan adquirir bienes y servicios cuando y donde lo necesitan" (p.256). Este proceso inicia con los proveedores de materias primas que llega al fabricante y este se encarga de generar distribución y de allí a los retailer hasta llegar a los compradores finales con este proceso se pretende generar niveles de ventas óptimos a través de diferentes estrategias basadas en ofertas y promociones publicitarias de los productos utilizando merchandising como una de las herramientas imprescindibles para lograr influir en el consumidor.

La empresa del Grupo el Rosado se enfoca en diferentes estrategias que oriente a las tres líneas de negocio cada línea aplica estrategias diferentes para cada línea por ejemplo en la retail de Mi Comisariato aplica estrategias de visibilidad de productos basándose en la presentación física del producto en las estanterías generando en los clientes atracción al momento de comprar. Romero y Salas (2018) describe que las estrategias de gestión de categorías de productos permiten desarrollar promociones para captar la atención de los clientes esto hacen las empresas cuando un stop de productos no tiene rotación. Otra de las estrategias que utilizan en el retail son modificar las generaciones de producto y en las perchas se transforma en un fuerte contingente para realizar cambios con los 
productos que no tienen rotación o también cuando hay un producto nuevo en el mercado.

$\mathrm{Al}$ ser una empresa con cadenas de supermercados tiene como estrategias realizar activaciones en lugares específicos con nuevos productos que penetran el mercado, estos lo aplican con degustaciones de los principales productos. Monferrer (2013) caracteriza. “En esta estrategia la determinación de compra es fundamental para la empresa y se basa en las diferentes actitudes que puede tener el cliente al momento de degustar un producto, aquí las influencias que tienen los otros compradores son un componente básico para comprar el producto" (p.87).

Otras estrategias que utiliza esta empresa son basadas en la planificación estratégica de segmentación basada en los comportamientos de compra que tiene los consumidores, "Para que el segmento de mercado resulte atractivo debe ser accesible. El primer requisito es que a la empresa le resulte fácil el acceso a canales que permitan poner un producto a disposición de los clientes finales" (Best, 2017, p.148). El crecimiento vertiginoso que ha tenido es porque su enfoque está en las necesidades que tienen el consumidor para inducir a la compra. La introducción de diferentes líneas al mercado establece un beneficio al consumidor al instante de comprar y que todos los productos estén al alcance en un solo lugar. Lo que se pretende por ejemplo es que en un hipermarket encuentren absolutamente todos los productos que necesita el cliente desde víveres, ferretería, entretenimiento, restaurante, esto permite al cliente cubrir todas las necesidades en un solo lugar teniendo a su alcance una gama de productos y servicios. (Staton, Etzel, y Walker, 2007, p.149)

\section{Herramientas en el proceso del trade marketing en la empresa Grupo el Rosado}

Una de las principales herramientas que utiliza esta empresa como estrategia basadas en un calendario anual que establecen promociones durante el ańo, por ejemplo utiliza cuponeras que al instante de comprar le dan un stiker y con eso podrá llenar la cuponera, el cliente obtendrá premios a cambio del llenado, también genera promociones por órdenes de comprar mayor a \$25 le dan un cupón para el sorteo de un carro, esta promoción la hacen para fechas como navidad, día de la madres. Otra forma de esta- 
blecer relación con los clientes es basada en tarjetas que utilizan para ganar puntos por comprar y descuentos al presentar la tarjeta, estas estrategias resultan significativas para los consumidores al momento de comprar. “ Las promociones consisten en que los fabricantes se dirigen a los intermediarios con actividades promocionales para empujar sus productos a través de canales mediante el incremento de sus ventas y alentando un mayor esfuerzo entre los socios del canal" (Ferre y Hartline, 2012, p.317).

Otra herramienta que se utiliza Grupo el Rosado en el trade marketing es el Merchandising que con los años ha tenido un proceso de desarrollo y evolución en el mercado, el uso de esta herramienta es fundamental ya que permite que la presentación del producto en el establecimiento para atraer a los clientes a comprar. Los ambientes creados en los establecimientos son fundamentales para los clientes se sienta atraído al momento de comprar esta estrategia tienen como propósito mejorar la asistencia de los clientes al establecimiento y así obtener mayor posibilidad para la compra (Ángel y Fajardo, 2016).

Las principales características del merchandising son los espacios tangibles e intangibles en los puntos de ventas, se fundamenta en la presentación del producto basado en esquemas promocionales como los elementos comunicativos y visuales para optimizar las diferentes técnicas de ventas que generen mayor fuerza en el mercado. (Fernández y González, 2017). En si el trade marketing es una herramienta que permite utilizar estrategias que faciliten a los clientes obtener las necesidades básicas como las fisiológicas, seguridad, sociales, estima y autorrealización que Maslow la describe como básicas en el desarrollo del ser humano. Viendo desde esta perspectiva Grupo el Rosado con sus líneas de negocio a desarrollo su estructura comercial para satisfacer todas las necesidades de los clientes.

\section{El ECR como proveedor eficiente del consumidor en la empresa Grupo el Rosado}

La respuesta eficiente al consumidor (ECR) forma un enfoque significativo que tiene la empresa en especial los supermercados la cual permite ganar cuota en el mercado ya que permite minimizar gastos innecesarios que tienen estás empresas. Sus orígenes se relacionan por el crecimiento 
comercial e industrial, después lo adapta a las empresas como los supermercados generando niveles óptimos de eficiencia en todo el contexto que se genera la distribución de los diferentes productos que tiene la empresa. El proceso tecnológico se da constantemente en el comercio permite integrar diferentes procesos que se dan entre los proveedores, distribuidores y detallista, está interrelación unificada hace que las operaciones generen eficiencia en la entrega de los productos y servicios.

El desarrollo tecnológico del ECR permite generar diferentes códigos de barras que permiten identificar a los productos con dígitos que identifican el código del país y también en un sistema es un sistema que integra la logística de los productos hasta llegar al consumidor final de la empresa Grupo el Rosado esta empresa establece una cadena de distribución como centro de logística en la ciudad de Guayaquil donde distribuye a las diferentes ciudades vía terrestre, cuando recibe productos importados también utilizan un sistema para dar seguimiento de sus productos que en su mayoría importa de china y EE.UU. así como también tienen sus propias fábricas de productos como la marca TEKNO que es de audio y video y se distribuye en los diferentes establecimientos del país.

$\mathrm{Al}$ ser una empresa que maneja tres líneas principales de producto su enfoque esta dado en optimización de sus pedidos dentro de los establecimientos. Para que exista un abastecimiento controlado y eficiente para el consumo de los clientes el objetivo del ECR es generar una red de valor para esto primero se establece que mejore de manera eficiente el funcionamiento de la distribución y segundo hace referencia a optimizar los recursos que abaraten los costos de traslados de productos. (Bello y Camacho, 2016). Basado en lo que manifiesta los autores hay una interrelación entre la logística y el desarrollo comercial, estos dos factores establecen el abastecimiento de manera eficiente los productos para el reposicionamiento de los productos de consumo y el aspecto comercial se fundamenta en lograr el abastecimiento o surtido de productos, en esta concepción las ventas son su principal objetivo junto a crear una satisfacción y fidelidad en los clientes cuando consumen los productos o servicios que ofrece la empresa. Su principal enfoque que tiene la empresa es estar interconectados tanto con los proveedores, empresa y clientes generando conexiones entre los clientes para crear valor agregado que contribuya el desarrollo comercial. 


\section{Conclusiones.}

Como resultado de esta investigación del caso práctico con la empresa Grupo el Rosado que surgió como una pequeña pastelería y que en el transcurso de estos 83 años se ha desarrollado vertiginosamente pasando por todos los procesos comerciales y tecnológicos que siempre mantuvo un sistema de innovación a la vanguardia de las principales cadenas de supermercados en Latinoamérica y el mundo. En Ecuador ha sido pionera en el crecimiento económico del país estableciendo fuentes de trabajo en las principales ciudades del País.

El desarrollo comercial y tecnológico se ha basado en la aplicación de estrategias de marketing que con el pasar de los años ha evolucionado y se podría decir que esta empresa es pionera en Ecuador en procesos innovadores comerciales y ha creado marcas en tres líneas de negocio que son el retail, entretenimiento y restaurantes generando en los clientes satisfacción total en sus necesidades ya que pueden encontrar todo en un solo lugar. El trade marketing que utiliza esta empresa tiene como objetivo la logística y las ventas para generar una cadena de valor que este interrelacionada entre sí, estos procesos van de la mano con los avances tecnológicos que permiten distribuir los productos de manera eficiente a todos los establecimientos para que exista un reposicionamiento de los productos y servicios que comercializan en el Grupo el Rosado.

\section{Bibliografía}

Angel, A \& Fajardo, W. (2016). Diseño de estrategias de merchandasing para mejorar la imagen visual del supermercado EDYMOR del cantón Guayaquil, año 2016. (tesis de pregrado). Universidad de Guayaquil, Ecuador. Recuperado de http://repositorio.ug.edu.ec/

Bello, L \& Camacho, L. (2016). Propuesta metodológica de implementación: Mmodelo ECR para el mejoramiento y eficiencia de la red de valor del supermercado $1 A$ (tesis de posgrado). Universidad Piloto de Colombia. Colombia. Recuperado de http://polux.unipiloto.edu. co:8080/00003015.pdf

Best, R. (2007). Marketing Estratégico. Madrid: Pearson Educación. 
Carrillo, R. (2017). Trade Marketing. (tesis de pregrado). Universidad de Lima, Perú. Recuperado de http://repositorio.ulima.edu.pe/bitstream/handle/ulima/4821/Carrillo_Ricardo.pdf?sequence $=1 \&$ isAllowed $=\mathrm{y}$

Fernández, D., \& González, R. (2017). Técnicas básicas de merchandising. España: Ediciones Paraninfo S.A.

Ferrel, O., \& Hartiline, M. (2012). Estrategia de Marketing. México: Cengage Learning

Historia del Grupo el Rosado (s.f) Recuperado el 18 de diciembre del 2019, sitio web. https://www.elrosado.com/

Jobber, D., \& Lancaster, G (2012). Administración de Ventas. México: Pearson Educación.

Kotler, P., \& Armstrong, G. (2013). Fundamentos de Marketing. México: Pearson Educación.

Monferrer, D. (2013). Fundamentos de Marketing. España: Publicaciones de la Universidad de Jaume. Recuperado de http://repositori.uji. es/xmlui/bitstream/handle/10234/49394/s74.pdf

Romero, M., \& Salas, L (2018). Diseño de estrategias de trade marketing para la empresa Danec. (tesis de pregrado). Universidad de Guayaquil, Ecuador. Recuperado de http://repositorio.ug.edu.ec/bitstream/ redug/35489/1/Tesis\%20Melgar\%20y\%20Salas\%201\%20 DE\%20SEP.pdf

Staton, E., Etzel, M., \& Walker, B. (2007). Fundamentos de Marketing. México: McGraw-Hill Interamericana 